\title{
A CONSCIÊNCIA FONOARTICULATÓRIA EM CRIANÇAS COM DESVIO FONOLÓGICO
}

\section{Articulatory awareness in children with speech disorders}

\author{
Débora Vidor-Souza ${ }^{(1)}$, Helena Bolli Mota ${ }^{(2)}$, Rosangela Marostega Santos ${ }^{(3)}$
}

\begin{abstract}
RESUMO
Objetivo: verificar as habilidades em consciência fonoarticulatória de crianças com desvio fonológico, comparando-as com as habilidades em consciência fonoarticulatória de crianças com desenvolvimento fonológico normal. Método: participaram do estudo 60 crianças, sendo 30 do grupo controle, com desenvolvimento fonológico normal e 30 do grupo estudo, com desvio fonológico. Todos os participantes foram submetidos à avaliação da consciência fonoarticulatória e os desempenhos dos dois grupos foram comparados. Resultados: pode-se verificar diferença estatisticamente significante entre o grupo controle e o grupo estudo nas tarefas de consciência fonoarticulatória, com maiores escores para o grupo controle. Conclusões: crianças com desvio fonológico apresentam maior dificuldade em habilidades de consciência fonoarticulatória se comparadas a crianças com desenvolvimento fonológico normal.
\end{abstract}

DESCRITORES: Distúrbios da Fala; Percepção da Fala; Criança

\section{INTRODUÇÃO}

Consciência fonológica é a habilidade que permite ao ser humano pensar e agir sobre os sons da fala ${ }^{1,2}$. Essa habilidade possui diferentes níveis: a consciência da palavra, da sílaba, das unidades intrassilábicas e do fonema ${ }^{3}$.

Esses níveis se aprimoram espontaneamente ou por meio do ensino formal da linguagem escrita, dependendo da sua complexidade ${ }^{4}$. O desenvolvimento dos níveis de consciência fonológica ocorre em etapas sucessivas, integrando um processo continuado, não necessariamente linear ${ }^{5}$.

(1) Fonoaudióloga; Mestranda do Programa de Pós-Graduação em Distúrbios da Comunicação Humana na Universidade Federal de Santa Maria, UFSM, Santa Maria, RS, Brasil.

(2) Fonoaudióloga; Professora Associada do Curso de Fonoaudiologia e do Programa de Pós-Graduação em Distúrbios da Comunicação Humana da Universidade Federal de Santa Maria, UFSM, Santa Maria, RS, Brasil; Doutora em Linguística Aplicada pela Pontifícia Universidade Católica do Rio Grande do Sul, PUCRS.

(3) Fonoaudióloga; Professora da Pós-Graduação em Alfabetização e Psicopedagogia da Faculdade Porto Alegrense de Educação Ciências e Letras, FAPA/POA, Porto Alegre, RS, Brasil; Mestre em Linguística Aplicada pela Pontifícia Universidade Católica do Rio Grande do Sul, PUCRS.

Conflito de interesses: inexistente
A consciência articulatória é parte da consciência fonológica e representa a capacidade de o falante refletir sobre as características articulatórias dos fonemas, podendo assim contribuir para a sua representação ${ }^{6}$.

Consciência fonoarticulatória é a habilidade responsável pela distinção das articulações dos sons da fala. É a capacidade do indivíduo pensar sobre os sons e relacioná-los aos movimentos que os articuladores fazem para produzi-los. Essa habilidade, além de auxiliar a percepção e produção da fala, facilita a aprendizagem do sistema de escrita alfabético ${ }^{7}$.

Uma pesquisa que teve como objetivo de relacionar a consciência fonoarticulatória e a linguagem escrita revelou que o desempenho em consciência fonoarticulatória das crianças do sexo feminino é superior ao masculino e que existe relação entre a consciência fonoarticulatória e a aquisição da linguagem escrita. Com isso, as autoras do estudo inferem que pensar sobre os sons da fala e os movimentos que os articuladores fazem para produzi-los é uma habilidade facilitadora para a aprendizagem do sistema alfabético de escrita ${ }^{8}$.

Desvios fonológicos são alterações linguísticas que se manifestam pelo uso de padrões anormais na produção da fala ${ }^{9}$. 
A criança com desvio fonológico apresenta algumas características clássicas, tais como: idade superior a quatro anos, fala espontânea desviando da pronúncia adulta alvo e audição normal para a fala. Além disso, a criança não deve apresentar qualquer anormalidade anatômica ou fisiológica nos mecanismos de produção da fala ou qualquer disfunção neurológica. Suas capacidades intelectuais devem estar adequadas para o desenvolvimento da linguagem, apresentando compreensão da linguagem e linguagem expressiva aparentemente normal ${ }^{9}$.

Desvio fonológico é uma dificuldade específica da linguagem que afeta a produção da fala, sem fatores etiológicos detectáveis ${ }^{10}$. No desvio fonológico o obstáculo encontra-se exatamente na dificuldade de organização mental, de estabelecimento do sistema fonológico da língua-alvo, de adequação ao input recebido. Esses desvios são de etiologia desconhecida, embora haja grande número de trabalhos que procuram possíveis fatores influentes ${ }^{11}$.

Avaliando as habilidades fonológicas de crianças com aquisição de linguagem normal, é possível perceber indícios de que elas possuem melhor conhecimento da fonologia da língua do que podem representar nas suas produções ${ }^{11}$. Um estudo do qual participaram crianças com desvio fonológico submetidas a uma avaliação de consciência fonológica, mostrou que as crianças com desvios fonológicos, da mesma forma que as crianças com aquisição de linguagem normal, parecem possuir habilidades fonológicas superiores às suas produções ${ }^{12}$.

No entanto, outros autores afirmam que o desempenho em consciência fonológica das crianças com desvio fonológico é inferior se comparado ao desempenho das crianças com sistema fonológico normal, mesmo quando as crianças com desvio fonológico apresentam nível cognitivo mais avançado ${ }^{13}$.

De acordo com a literatura atual, os desvios fonológicos parecem estar relacionados às dificuldades em consciência fonológica. Um estudo que teve por objetivo avaliar a relação entre os desvios fonológicos e o desenvolvimento das habilidades em consciência fonológica, comparando um grupo de crianças com desvios fonológicos a outro composto por crianças com desenvolvimento fonológico normal. As autoras confirmaram um desempenho inferior em provas de consciência fonológica por parte das crianças com desvio fonológico em relação às crianças com desenvolvimento fonológico normal ${ }^{14}$.

Crianças com desvios fonológicos são consideradas de risco para a presença de futuras dificuldades no desenvolvimento da linguagem, portanto, salienta-se a importância de estimular as habilidades em consciência fonológica, não somente em crianças na fase pré-escolar, mas principalmente em crianças com desvio fonológico ${ }^{15-21}$.

Um estudo, com 11 pré-escolares, formado por um grupo de sujeitos com atraso de linguagem e/ ou desvio fonológico e outro grupo de crianças com desenvolvimento normal, concluiu que os participantes com atraso de fala e/ou desvio fonológico apresentaram dificuldades nas habilidades em consciência fonológica. Os autores sugerem que tais alterações podem ser minimizadas por meio de um programa de intervenção em habilidades em consciência fonológica ${ }^{22}$.

Foi realizada uma pesquisa investigando a aplicabilidade de um modelo terapêutico que enfoca o uso da consciência fonológica, o Metaphon, como fundamento para o tratamento de crianças com desvio fonológico. Os achados evidenciaram a efetividade de tal abordagem ao permitir o estabelecimento dos contrastes fonológicos da Língua Portuguesa Brasileira na fala de crianças com desvio fonológico, mediante a generalização estrutural e funcional ${ }^{23}$.

Outro estudo que teve por objetivo confrontar o desempenho das habilidades em consciência fonológica de um grupo de sujeitos com desenvolvimento fonológico normal com indivíduos com histórico de transtorno fonológico, após sua superação, por meio de terapia fonológica, avaliou 18 sujeitos. Os grupos apresentaram diferenças estatisticamente significantes em seus desempenhos em todas as atividades de consciência fonológica, sendo que o grupo composto pelos sujeitos com desenvolvimento fonológico normal obteve melhor desempenho. Com base nisso, as autoras concluíram que mesmo após a intervenção fonológica o grupo dos indivíduos com histórico de transtorno fonológico apresentou desempenho inferior nas habilidades de consciência fonológica ${ }^{24}$.

Foi desenvolvido um estudo que teve por objetivo analisar a relação entre as habilidades em consciência fonoarticulatória e o uso da estratégia de alongamento compensatório em três crianças com desvio fonológico. Considerando que o uso de tal estratégia pressupõe que a criança possui determinado fonema na subjacência, porém não consegue produzi-lo, infere-se que ela demonstra melhor reconhecimento fonético dos sons comparado a crianças que não fazem uso da estratégia de alongamento compensatório. As autoras puderam inferir que as habilidades em consciência fonoarticulatória possuem relação com o uso da estratégia de alongamento compensatório ${ }^{25}$. 
Acredita-se que o apoio fonoarticulatório é de suma importância no processo de aquisição e desenvolvimento dos sons da fala e que este é um recurso utilizado de forma implícita na clínica fonoaudiológica. Além disso, sabe-se que conhecer o desempenho de crianças com desvios fonológicos em tarefas envolvendo habilidades fonológicas, incluindo as habilidades fonoarticulatórias, é fundamental, pois auxilia na compreensão do distúrbio de linguagem e contribui para a elaboração dos programas de reabilitação ou de estimulação dessas habilidades considerando as necessidades individuais.

Com base na literatura atual, que evidencia que sujeitos com desvio fonológico possuem dificuldades em atividades envolvendo habilidades em consciência fonológica, realizou-se um estudo com o objetivo de verificar as habilidades em consciência fonoarticulatória de crianças com desvio fonológico, comparando-as às habilidades em consciência fonoarticulatória de crianças com desenvolvimento fonológico normal.

\section{MÉTODO}

O presente estudo é transversal e observacional. Os fatores em estudo foram a consciência fonoarticulatória e o desvio fonológico. E o desfecho foi a relação entre eles.

Este estudo está incluído no projeto intitulado "A estratégia de alongamento compensatório e sua relação com a performance nas habilidades de consciência fonológica".

Participaram dessa pesquisa quatro instituições, duas escolas públicas e uma filantrópica e o serviço de atendimento fonoaudiológico de uma universidade, onde se desenvolve este projeto, foram contatadas com o intuito de esclarecer os objetivos da pesquisa e obter o Consentimento Institucional.

Após a obtenção dos Consentimentos Institucionais e a aprovação deste projeto no Comitê de Ética em Pesquisa, foi entregue aos pais/responsáveis dos prováveis participantes o Termo de Consentimento Livre e Esclarecido no qual constam informações sobre os objetivos da pesquisa, os procedimentos a serem adotados, riscos e benefícios, sendo a assinatura desse documento e o assentimento da criança condições para a participação da criança na pesquisa.

Nas escolas, marcou-se, por intermédio dos professores, uma reunião com os pais de todas as crianças com a idade adequada para participar da pesquisa. Nesta reunião foram esclarecidos os objetivos e procedimentos da pesquisa com a entrega do Termo de Consentimento Livre e Esclarecido. Com todas as crianças que concordaram em participar da pesquisa e cujos pais assinaram o Termo de Consentimento Livre e Esclarecido foi realizada a triagem fonoaudiológica, composta por triagem auditiva, avaliação dos órgãos fonoarticulatórios, avaliação da linguagem compreensiva e expressiva oral e avaliação dos sistemas fonético e fonológico. Já as avaliações para a coleta de dados foram realizadas somente com as crianças que preencheram os critérios para inclusão dos sujeitos na amostra.

No serviço de atendimento fonoaudiológico, os sujeitos foram selecionados por meio das triagens do setor de fala nas quais já constam algumas informações como a presença de trocas na fala e quais trocas são produzidas pelo sujeito. Com isso, os pais/responsáveis foram contatados e convidados a participar da pesquisa. Após ser consentida a participação na mesma foram realizadas com os indivíduos todas as avaliações que não faziam parte da triagem do serviço de atendimento fonoaudiológico e que foram necessárias para a composição da amostra.

A amostra foi composta pelos sujeitos selecionados a partir das triagens do setor de fala do serviço de atendimento fonoaudiológico e em três escolas. Os sujeitos da amostra foram divididos em dois grupos: o grupo com desvio fonológico (grupo estudo) e o grupo com desenvolvimento fonológico normal (grupo controle).

Para a seleção da amostra foram estabelecidos os seguintes critérios de inclusão: os sujeitos deveriam ter idades entre cinco a sete anos onze meses e vinte e nove dias, não estar em terapia fonoaudiológica e os pais e ou responsáveis deveriam assinar o Termo de Consentimento Livre e Esclarecido.

Os sujeitos da amostra foram submetidos a uma triagem fonoaudiológica que se constituiu das seguintes avaliações: triagem auditiva, avaliação dos órgãos fonoarticulatórios, avaliação da fala e avaliação da linguagem compreensiva e expressiva oral, conforme a boa prática clínica.

$\mathrm{Na}$ avaliação dos órgãos fonoarticulatórios foram realizados exames intra e extra-orais. Foram avaliadas as estruturas moles (língua, lábios e bochechas) e as estruturas duras (dentes e estruturas ósseas). No que se refere às funções do sistema estomatognático foram avaliadas respiração, mastigação e deglutição. Esta avaliação se fez necessária para que fosse descartado qualquer comprometimento nos órgãos fonoarticulatórios que pudesse interferir na produção da fala.

Para a avaliação da linguagem foi apresentada uma sequência lógica de quatro fatos fora de ordem (ilustração de um aniversário) para a criança organizar e contar oralmente uma história além de responder questões a respeito das figuras. 
A história foi gravada e, posteriormente, transcrita para que se realizem as análises. Com esta avaliação buscou-se investigar se a linguagem compreensiva e expressiva oral estava adequada à idade da criança.

$\mathrm{Na}$ avaliação dos sistemas fonológico e fonético foi aplicado um instrumento constituído de cinco desenhos que possibilitam a obtenção de uma amostra de fala, por meio de nomeação e fala espontânea, contendo todos os fones contrastivos do Português em todas as posições que podem ocorrer em relação à estrutura da sílaba e da palavra ${ }^{26}$.

A triagem auditiva foi realizada com o audiômetro Interacoustics Screening Audiometer AS208, devidamente calibrado. Inicialmente as crianças foram submetidas à inspeção do meato acústico externo de cada uma das orelhas para verificar a presença de corpos estranhos ou excesso de cerúmem, que pudessem estar obstruindo a via aérea. Após, foi realizada a investigação dos limiares de via aérea nas frequências de $500 \mathrm{a} 4000 \mathrm{~Hz}$ testadas a $20 \mathrm{~dB}$. Vale ressaltar que tal avaliação foi realizada em ambiente silencioso. Qualquer alteração observada nesta avaliação implicaria no encaminhamento da criança ao médico otorrinolaringologista.

Foi estabelecido que seriam excluídas da amostra as crianças que apresentassem doenças neurológicas, crianças com deficiência mental, crianças com problemas psicológicos evidentes, crianças com queixas escolares de dificuldade de aprendizagem, crianças que falhassem na triagem auditiva, crianças que apresentassem alterações na avaliação dos órgãos fonoarticulatórios e crianças que apresentassem alterações de linguagem.

A população do estudo foi composta por 90 crianças com desenvolvimento fonológico normal, que formaram o grupo controle e 30 crianças com desvio fonológico para compor o grupo estudo. Optou-se por deixar o grupo controle com o mesmo número de sujeitos do grupo estudo. Para isso, foram pareados por sexo e idade os integrantes do grupo controle ao grupo estudo, para controlar a influência desses fatores na comparação dos escores.

Para poder parear os grupos em termos de sexo e idade, fixou-se cada subgrupo (Meninas cinco anos, Meninas seis anos, Meninas sete anos, Meninos cinco anos, Meninos seis anos e Meninos sete anos) do grupo estudo, e então foram sorteadas as crianças entre todas do grupo controle. A única exceção foi o grupo Meninos com cinco anos, que tinha sete crianças no grupo estudo, e apenas cinco no grupo controle. Neste caso foram sorteados mais dois meninos da próxima faixa etária, deixando assim os grupos homogêneos para sexo e idade.

A coleta de dados teve início no primeiro semestre de 2008, no mês de julho, e foi realizada nas escolas selecionadas e no serviço de atendimento fonoaudiológico.

Para a coleta dos dados foi utilizado instrumento de investigação "Consciência Fonoarticulatória" ${ }^{27}$ que busca avaliar as habilidades em consciência fonoarticulatória por meio de cinco tarefas, utilizando-se de seis fotografias de gestos fonoarticulatórios, a saber: boca $A$ correspondendo aos fones [p], [b] e [m]; boca B correspondendo aos fones [f] e [v]; boca C correspondendo ao fone [l]; boca D correspondendo aos fones [s] e [z]; boca E correspondendo aos fones []] ou [3] e boca F correspondendo aos fones $[\mathrm{k}] \mathrm{e}[\mathrm{g}]$.

Para cada uma das tarefas do teste foram apresentados dois exemplos, para que o examinador pudesse certificar-se de que a criança compreendeu a atividade, então a criança foi solicitada a responder quatro questões a serem pontuadas, totalizando vinte pontos no máximo.

Na primeira tarefa (T1) deve-se identificar a fotografia contendo o gesto fonoarticulatório correspondente ao som apresentado à criança. Por exemplo, ao ser produzido o [v], o sujeito deverá selecionar a figura da boca B. Para a realização da tarefa dois (T2) apresenta-se uma foto de boca realizando um gesto fonoarticulatório e a criança deve produzir o som correspondente a ele. Por exemplo, ao mostrar-se a boca $A$, o sujeito deverá produzir 0 [m]. A terceira tarefa (T3) consiste em identificar o gesto fonoarticulatório correspondente ao primeiro som de uma palavra apresentada de forma oral pelo examinador. Nessa tarefa pode-se utilizar o apoio visual, com fotos que ilustrem as palavras apresentadas. Por exemplo, ao ser apresentada a palavra carro, espera-se que a criança selecione a boca F. Na quarta tarefa (T4) é solicitado ao sujeito que identifique dentre quatro palavras, apresentadas em forma de fotografias, qual é a correspondente ao gesto fonoarticulatório. Por exemplo, ao ser apresentada a boca E, espera-se que o sujeito identifique a figura da chave. Na última tarefa (T5) a criança deve produzir uma palavra a partir de uma foto de boca que contenha um gesto fonoarticulatório que deverá corresponder ao primeiro som da palavra produzida. Por exemplo, ao ser apresentada a boca $\mathrm{C}$, a criança deverá dizer uma palavra que inicie com [l].

Essa pesquisa foi aprovada pelo Comitê de Ética em Pesquisa da Universidade Federal de Santa Maria, com cadastro sob número 0103/2007. 
Os resultados obtidos na coleta de dados foram tabelados e então foi realizada uma análise descritiva do grupo estudo por meio de tabelas de frequência e estatísticas descritivas (Tabela 1). Posteriormente foi realizada a análise comparativa do percentual de acertos e pontuação nas tarefas do Instrumento de Investigação da Consciência Fonoarticulatória entre os grupos controle e estudo por meio do teste Mann-Whitney. O nível de significância adotado para os testes estatísticos foi de $5 \%$, ou seja, $\mathrm{P}<0.05$.

Tabela 1 - Análise descritiva das variáveis para caracterização do grupo estudo

\begin{tabular}{ccc}
\hline \multicolumn{3}{c}{ IDADE } \\
\hline Idade & Frequência & Percentual \\
5 & 13 & 43.33 \\
6 & 11 & 36.67 \\
7 & 6 & 20.00 \\
\hline & SEXO & \\
\hline Sexo & Frequência & Percentual \\
M & 14 & 46.67 \\
\hline
\end{tabular}

\section{RESULTADOS}

Na Tabela 2 são apresentados os desempenhos do grupo controle e do grupo estudo por meiodas médias de acertos, desvio padrão, mínimo de acertos, mediana e máximo de acertos em cada uma das tarefas de consciência fonoarticulatória, e no escore total do instrumento.

Os resultados podem ser observados de forma ilustrativa na Figura 1 que mostra um gráfico com a comparação das médias e desvio padrão do grupo controle e do grupo estudo, obtidos em cada uma das tarefas de consciência fonoarticulatória. Pela análise do gráfico, é possível observar que os boxplots em azul (grupo controle) estão sempre mais acima que os em vermelho (grupo estudo), indicando melhor desempenho do grupo controle.

\section{DISCUSSÃO}

Os achados deste estudo apontam que as crianças com desvio fonológico apresentam desempenho inferior nas habilidades de consciência fonoarticulatória, se comparadas às crianças com desenvolvimento fonológico normal.

Por meio da análise dos resultados pode-se verificar diferença estatisticamente significante entre o grupo controle e o grupo estudo para as seguintes variáveis: escores das tarefas T1, T2, T3 e T5 e escore total do teste, com maiores escores para o grupo controle.

$\mathrm{Na} \mathrm{T} 1$, que envolve a identificação do gesto fonoarticulatório a partir do som, a média de acertos para o grupo controle foi 2.50 e para o grupo estudo foi 1.73. Já na T2, que consiste na produção do som a partir do gesto fonoarticulatório, o grupo controle

Tabela 2 - Comparação dos desempenhos em consciência fonoarticulatória entre o grupo controle e o grupo estudo

\begin{tabular}{|c|c|c|c|c|c|c|c|c|c|c|c|c|c|c|}
\hline \multicolumn{7}{|c|}{ GRUPO CONTROLE } & \multicolumn{8}{|c|}{ GRUPO ESTUDO } \\
\hline VARIÁVEL & $\mathbf{N}$ & MÉDIA & D.P. & MÍN & MEDIANA & MÁX & VARIÁVEL & $\mathbf{N}$ & MÉDIA & D.P. & MíN & MEDIANA & MÁX & VALOR-P* \\
\hline $\mathrm{T} 1$ & 30 & 2.50 & 1.41 & 0.00 & 2.00 & 4.00 & $\mathrm{~T} 1$ & 30 & 1.73 & 1.05 & 0.00 & 2.00 & 4.00 & $P=0.019$ \\
\hline T2 & 30 & 2.60 & 1.22 & 0.00 & 3.00 & 4.00 & T2 & 30 & 1.73 & 1.08 & 0.00 & 2.00 & 4.00 & $P=0.005$ \\
\hline T3 & 30 & 2.00 & 1.05 & 0.00 & 2.00 & 4.00 & T3 & 30 & 0.97 & 0.56 & 0.00 & 1.00 & 2.00 & $P<0.001$ \\
\hline $\mathrm{T} 4$ & 30 & 2.03 & 1.54 & 0.00 & 2.00 & 4.00 & T4 & 30 & 1.63 & 1.54 & 0.00 & 1.00 & 4.00 & $P=0.294$ \\
\hline T5 & 30 & 2.00 & 1.51 & 0.00 & 2.00 & 4.00 & T5 & 30 & 1.00 & 1.17 & 0.00 & 1.00 & 4.00 & $P=0.009$ \\
\hline TOTAL & 30 & 11.13 & 4.98 & 2.00 & 11.50 & 20.00 & TOTAL & 30 & 7.07 & 3.14 & 1.00 & 7.00 & 14.00 & $P<0.001$ \\
\hline
\end{tabular}

* Valor-P referente ao teste de Mann-Whitney para comparação das variáveis entre grupos (Controle vs Estudo). 


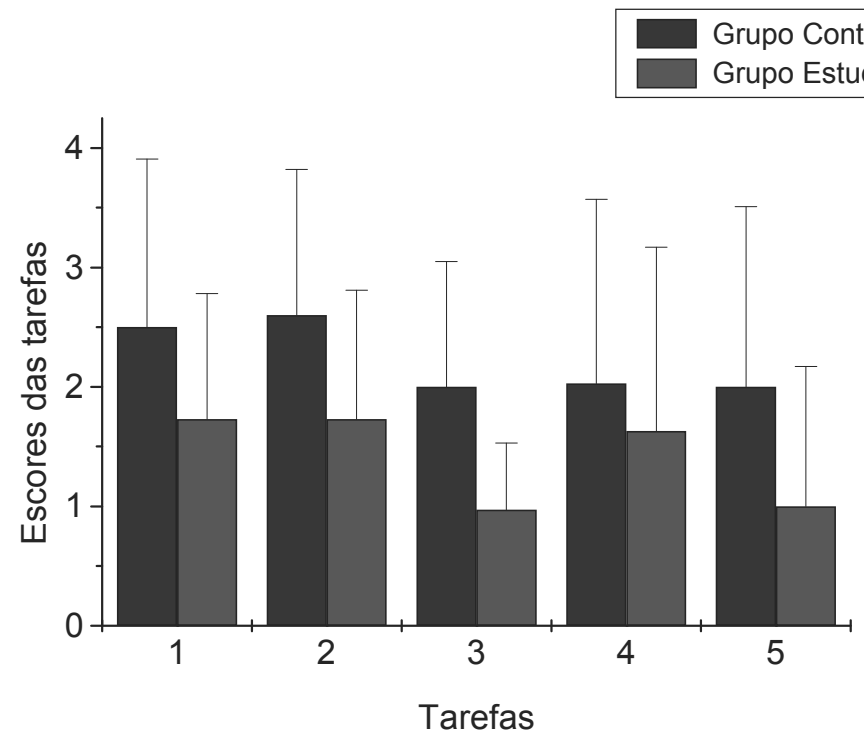

Figura 1 - Comparação dos desempenhos em consciência fonoarticulatória entre o grupo controle e o grupo estudo

obteve uma média de acertos de 2.60 e o grupo estudo uma média de acertos de 1.73. Na terceira tarefa (T3), que envolve a identificação do gesto articulatório a partir da palavra, o grupo controle apresentou um média de acertos de 2.00 enquanto que o grupo estudo apresentou 0.97 de média de acertos. Para a T5, que consiste na produção de palavra a partir do gesto fonoarticulatório, o grupo controle obteve média de acertos de 2.00 e o grupo estudo apresentou média de acerto de 1.00. No escore total do teste o grupo controle apresentou média de acertos de 11.13 e o grupo estudo alcançou 7.07 como média de acertos no teste de consciência fonoarticulatória.

A comparação entre os escores do grupo controle e do grupo estudo para a T4, que envolve a identificação de palavra a partir do gesto fonoarticulatório, por meio do apoio de figuras, não apresentou diferença estatisticamente significativa, apesar de o grupo controle apresentar média de acertos superior a média de acertos obtida pelo grupo estudo.

Os resultados deste estudo corroboram os achados de diversas pesquisas em consciência fonológica que afirmam que crianças com desvio fonológico quando comparadas com crianças com desenvolvimento fonológico normal apresentam, de forma geral, um desempenho inferior em tarefas de consciência fonológica 13,14,21,22,28,29. Isso reforça a afirmação de que a consciência fonoarticulatória é parte importante da consciência fonológica ${ }^{7} \mathrm{e}$ que essa habilidade deve ser incluída nas rotinas de avaliação e tratamento de crianças com desvio fonológico.
Crianças com desvio fonológico possuem grande risco de apresentarem dificuldades nas habilidades em consciência fonológica se elas apresentarem pouca habilidade de percepção da fala e/ou vocabulário receptivo relativamente pobre. Portanto, essas crianças deveriam receber tratamento para a percepção da fala, vocabulário receptivo e consciência fonológica ${ }^{28}$. Da mesma forma, as crianças com desvio fonológico obtiveram desempenhos inferiores nas habilidades em consciência fonoarticulatória, o que leva a crer que o trabalho com tais habilidades também pode auxiliar no tratamento dos desvios fonológicos.

Um estudo que teve por objetivo investigar o desempenho de habilidades em consciência fonológica e memória de trabalho em crianças em idade pré-escolar com desvio fonológico mostrou que essas crianças apresentam pior desempenho nas tarefas de consciência fonológica e memória de trabalho, quando comparadas as crianças com desenvolvimento fonológico normal. Além disso, o estudo apontou que as crianças com desvio fonológico mais severo, apresentam maior dificuldade nas tarefas de consciência fonológica do que as crianças com menos alterações de fala ${ }^{28}$.

As crianças com dificuldades na fala, frequentemente apresentam atraso no desenvolvimento da consciência fonológica, associado à dificuldade no aprendizado da linguagem escrita. E ainda afirmam que a consciência fonológica é uma habilidade fundamental na terapia dos desvios de fala. Levando em consideração os resultados obtidos neste estudo, a consciência fonoarticulatória, da 
mesma forma, deve ser estimulada na terapia dos desvios de fala ${ }^{30}$.

As dificuldades apresentadas nas habilidades em consciência fonológica por crianças com desvio fonológico podem ser explicadas pelo tipo de conhecimento linguístico que tais sujeitos possuem. Por apresentarem representações fonológicas diferentes, as crianças com desvio fonológico tendem a dar respostas incorretas para as tarefas linguísticas, mesmo que elas possuam acesso ao conhecimento fonológico e capacidade para análise semelhante às crianças com desenvolvimento fonológico normal ${ }^{31}$. O fato de as crianças com desvio fonológico apresentarem representações fonológicas diferentes às de crianças com desenvolvimento normal de fala, também pode ser considerada a explicação para o desempenho inferior das crianças com desvio fonológico em habilidades de consciência fonoarticulatória. Uma vez que tal habilidade é considerada parte da consciência fonológica e tem apresentado em alguns estudos resultados semelhantes aos de pesquisas envolvendo a consciência fonológica ${ }^{8,25}$.

A partir dos estudos citados, é possível afirmar que as crianças demonstram mais facilidade para perceber que as palavras são formadas por fonemas quando atentam para o gesto fonoarticulatório. Por essa razão, infere-se que o apoio fonoarticulatório seja um facilitador no momento em que o sujeito aprende a associar fonemas com suas represen- tações gráficas, apoio esse que se torna implícito após a automatização dessa relação.

Esta pesquisa é um forte indicador de que crianças com desvio fonológico possuem rebaixadas as habilidades de consciência fonoarticulatória. No entanto, é importante ressaltar que este estudo foi realizado com um número pequeno de crianças e que os achados não podem ser generalizados. É necessário um estudo correlacionando as habilidades em consciência fonológica com as habilidades em consciência fonoarticulatória de crianças com desvio fonológico.

\section{CONCLUSÃO}

Crianças com desvio fonológico apresentam maior dificuldade em habilidades de consciência fonoarticulatória se comparadas a crianças com desenvolvimento fonológico normal.

Numa abordagem linguística contemporânea, a intervenção fonoaudiológica para os desvios de fala, especificamente, os de natureza fonológica, costuma envolver primariamente tarefas partindo da palavra. Com base nos resultados do presente estudo, salienta-se a importância de juntamente com essa abordagem realizar atividades envolvendo fonemas isolados, ou seja, enfatizar a realização fonética dos sons da fala que se encontram alterados para, posteriormente, inseri-los na palavra.

\begin{abstract}
Purpose: to check the articulatory awareness skills of children with speech disorders, comparing them to children with normal phonological development. Method: the study involved sixty children, thirty from the control group with normal phonological development and thirty from the study group with speech disorders. All subjects underwent assessment of articulatory awareness and we compared the performance of both groups. Results: data show a statistically significant difference between control and study group in the tasks concerning articulatory awareness, with higher scores for the control group. Conclusion: children with speech disorders have more difficulty in articulatory awareness skills when comparing their performance to that of children with normal phonological development.
\end{abstract}

KEYWORDS: Speech Disorders; Speech Perception; Child 


\section{REFERÊNCIAS}

1. Cielo CA. Avaliação de habilidades em consciência fonológica. J Bras Fonoaudiol. 2003; 4(16):163-74.

2. Cupples $L$, lacono T. Phonological awareness and oral reading skill in children with Down syndrome. J Speech Lang Hear Res. 2000; 43:595-608.

3. Oliveira CM. A apropriação do princípio alfabético: compreensão do processo. Rev Virtual de Estudos da Linguagem. 2005; 3(5). [acesso em dez 2006]. Disponível em: http://www.revelhp.cjb.net.

4. Capovilla AGS, Dias NM, Montiel JM. Desenvolvimento dos componentes da consciência fonológica no ensino fundamental e correlação com a nota escolar. Psico-USF. 2007; 12(1):55-64.

5. Gindri G, Keske-Soares M, Mota HB. Memória de trabalho, consciência fonológica e hipótese de escrita. Pró-Fono. 2007; 19(3):313-22.

6. Godard L. Troubles du langage écrit chez les enfants: dyslexie. Fréquences. 2007; 18(4):12-6.

7. Santos RM. Sobre consciência fonoarticulatória. In: Lamprecht R, Blanco-Dutra AP, Scherer APR, Barreto FM, Brisolara LB, Santos RM, Alves UK. Consciência dos sons da língua: subsídios teóricos e práticos para alfabetizadores, fonoaudiólogos e professores de língua inglesa. Porto Alegre: Edipucrs; 2009. 352p.

8. Vidor-Souza D, Santos RM. Relação entre consciência fonoarticulatória e linguagem escrita. In: Anais do $1^{\circ}$ Congresso Brasileiro de Fonoaudiologia e $7^{0}$ Congresso Internacional de Fonoaudiologia; 2007. Sociedade Brasileira de Fonoaudiologia; 2007. Gramado. [CD-ROM].

9. Grunwell P. The nature of phonological disability in children. Londres: Edward Arnold; 1981.

10. Mota HB. Terapia fonoaudiológica para os desvios fonológicos. Rio de Janeiro: Revinter; 2001. 11. Lamprecht RR. Antes de mais nada. In: Aquisição fonológica do Português: perfil de desenvolvimento e subsídios para a terapia. (org.). Aquisição fonológica do Português: perfil de desenvolvimento e subsídios para a terapia. Porto Alegre: Artmed; 2004. p. 17-32.

12. Menezes G, Lamprecht RR. A consciência fonológica na relação fala-escrita em crianças com Desvio Fonológico Evolutivo (DFE). Letras de Hoje. 2001; 36(3):743-9.

13. Major EM, Bernhardt BH. Metaphonological skills of children with phonological disorder before and after phonological and metaphonological intervention. Int $\mathrm{J}$ Lang Commun Disord. 1998; 4(33):413-44.

14. Morales MV, Mota HB, Keske-Soares M. Consciência fonológica: desempenho de crianças com e sem desvios fonológicos evolutivos. Pró-Fono. 2002a; 14(2):153-64.

15. Bernhardt B, Major E. Speech, Language and literacy skills 3 years later: a follow-up study of early phonological. Int J Lang Commun Disord. 2005; 40(1):1-27.

16. Paula GR, Mota HB, Keske-Soares M. A terapia em consciência fonológica no processo de alfabetização. Pró-Fono. 2005; 17(2):175-84.

17. Gillon GT. Facilitating phoneme awareness development in 3-and 4-year-old children with speech impairment. Lang Speech Hear Serv Schools. 2005; 36:308-24.

18. Denne M, Langdown N, Pring T, Roy P. Treating children with expressive phonological disorders: does phonological awareness therapy work in the clinic? Int J Lang Commun Disord. 2005; 40(4):493-504.

19. Moriarty BC, Gillon GT. Phonological awareness intervention for children with childhood apraxia of speech. Int J Lang Commun Disord. 2006; 41(6):713-34

20. Mota HB, Melo Filha MGC, Lasch SS. A consciência fonológica e o desempenho na escrita sob ditado de crianças com desvio fonológico após realização de terapia fonoaudiológica. Rev. CEFAC. 2007; 9(4):477-82.

21. Rvachew S, Chiang PY, Evans N. Characteristics of speech errors produced by children with and without delayed phonological awareness skills. Lang Speech Hear Serv Schools. 2007; 38(1):60-71.

22. Laing SP, Espeland W. Low intensity phonological awareness training in a school classroom for children with communication impairments. J Commun Disord. 2005; 38(1):65-82.

23. Ardenghi LG, Mota HB, Keske-Soares M. A terapia Metaphon em casos de desvios fonológicos. Rev Soc Bras Fonoaudiol. 2006; 11(2):106-15.

24. Mota HB, Melo Filha MGC. Habilidades em consciência fonológica de sujeitos após realização de terapia fonológica. Pró-Fono. 2009; 21(2):119-24.

25. Vidor-Souza D, Dias RF, Mota HB, Mezzomo CL. $\mathrm{O}$ uso da estratégia de alongamento compensatório versus habilidades em consciência fonoarticulatória em crianças com Desvio Fonológico Evolutivo. In: Anais do $16^{\circ}$ Congresso Brasileiro de Fonoaudiologia. 2008. Sociedade Brasileira de Fonoaudiologia; 2008. Campos do Jordão.

26. Yavas M, Hernandorena CLM, Lamprecht RR. Avaliação Fonológica da Criança: reeducação e terapia. Porto Alegre: Artmed, 1991.

27. Santos R, Vieira MJB, Vidor-Souza D. Consciência fonoarticulatória: instrumento de investigação. Porto Alegre: Farfalla Editora; 2008. Material em preparação para publicação. 
28. Vieira MG, Mota HB, Keske-Soares M. Memória de trabalho e consciência fonológica no desvio fonológico. [dissertação] Santa Maria (RS): Universidade Federal de Santa Maria; 2005.

29. Rvachew S, Grawburg M. Correlates of phonological awareness in preschoolers whit speech sound disorders. J Speech Lang Hear Res. 2006; 49:74-87.
30. Stackhouse J, Wells B, Pascoe M, Rees R. From phonological therapy to phonological awareness. Semin Speech Lang. 2002; 1(23):27-42.

31. Sutherland D, Gillon GT. Development of phonological representations and phonological awareness in children with speech impairment. Int $\mathrm{J}$ Lang Commun Disord. 2007; 42(2):229-50.

DOI: 10.1590/S1516-18462010005000115

RECEBIDO EM: 20/12/2009

ACEITO EM: 30/04/2010

Endereço para correspondência:

Débora Vidor-Souza

Av. Borges de Medeiros, 356

Garibaldi - RS

CEP: 95720-000

E-mail: debi.vs@gmail.com 